\title{
A Case of Alveolar Echinococcosis Occurring in the Hilar Bile Duct
}

\author{
Jinyu Yang', Zhanxue Zhao ${ }^{1, *}$, Shuai Li', Hekai Chen ${ }^{3}$ \\ 'Department of General Surgery, Qinghai Provincial People's Hospital, Qinghai Xining 810007, China; '2Department of Clinical Pharmacy, Affiliated \\ Hospital of Qinghai University, Qinghai Xining 810001, China; ${ }^{3}$ Department of General Surgery, Tianjin Fifth Central Hospital, Tianjin 300450, China
}

\begin{abstract}
Echinococcosis is a disease caused by the Echinococcus species that parasitizes in humans. Alveolar echinococcosis (AE) which is caused by Echinococcus multilocularis is harmful to humans. AE mainly occurs in the liver and can be transferred to retroperitoneal lymph nodes, lung, brain, bone, spleen and other organs through lymphatic and blood vessels. Cholangiocarcinoma can occur in the intrahepatic and extrahepatic bile ducts and is more common in the hilar. We reported a case of hilar bile duct alveolar echinococcosis which was originally misdiagnosed an cholangiocarcinoma.
\end{abstract}

Key words: Alveolar echinococcosis, hepatic, cholangiocarcinoma

\section{INTRODUCTION}

Hepatic echinococcosis is a zoonotic parasitic disease that is prevalent in the Mediterranean area, South America, Australia, Central Asia, and Northwestern China [1]. The larvae of the Echinococcus species include 6 species: E. granulosus, E. multilocularis, E. vogeli, E. oligarthrus, E. felidis, and E. shiquicus [2]. Alveolar echinococcosis (AE) caused by E. multilocularis, which mainly occurs in the liver, is the most harmful echinococcosis in human. Hepatic alveolar echinococcosis (HAE) is a diffuse and infiltrating-growth disease that can metastasize to retroperitoneal lymph nodes, lung, brain, bone, spleen and other organs through lymphatic vessels and blood vessels [3]. Although it is benign, its biological behavior is malignant, so it is also known as "worm cancer" and "second cancer" [4].

Alveolar echinococcosis rarely occurs in extrahepatic bile ducts. We reported a rare case of alveolar echinococcosis that occurred in the hilar bile duct that is differentiated from cholangiocarcinoma in this article.

- Received 22 November 2018, revised 26 July 2019, accepted 16 September 2019.

*Corresponding author (zhaozhanxue1025@sina.com)

(c) 2019, Korean Society for Parasitology and Tropical Medicine

This is an Open Access article distributed under the terms of the Creative Commons Attribution Non-Commercial License (http://creativecommons.org/licenses/by-nc/4.0) which permits unrestricted non-commercial use, distribution, and reproduction in any

medium, provided the original work is properly cited.

\section{CASE RECORD}

A 58-year-old Han Chinese woman visited to general surgery department of Qing Hai Provincial People's Hospital with chief complaints of pigastric pain for 3 months and the pain aggravated for 10 days. In addition, the patient also had symptoms of jaundice, nausea and vomiting. The patient had lived in Dari County for the 10 past years, which is the area with the highest incidence of echinococcosis in China, and she had been in close contact with cattle, sheep and dogs. She was diagnosed as hypertension 3 years ago. On physical examination, jaundice and pressing pain over the right upper quadrant were observed. MRI indicated cholecystitis, a right hepatic duct stone, intrahepatic bile duct dilatation, hepatic hilar bile duct truncation and left supra-renal abscess. Laboratory tests showed that the results of blood routine were normal, but the liver function and tumor markers were abnormal (Tables 1-3). CT scan suggested significant expansion of the intrahepatic bile ducts; multiple enlarged retroperitoneal lymph nodes; calcification in right lobe of liver and hilar malignant tumors (Figs. 1, 2). The upper abdominal MRI scan and MRCP revealed tumorous lesions of the upper end of common bile duct and obvious dilatation of intrahepatic and extrahepatic bile ducts (Fig. 3). We performed liver protection and rehydration symptomatic treatments as well as percutaneous transhepatic choledochic drainage (PTCD). According to relevant imaging 63 examinations and tests, we diagnosed the patient as 
Table 1. Results of routine blood test

\begin{tabular}{lccc}
\hline Project name & Result & Unit & Reference range \\
\hline White-cell count & 4.9 & $10^{9} / \mathrm{L}$ & $3.5-9.5$ \\
Hemoglobin level & 136 & $\mathrm{~g} / \mathrm{L}$ & $119-157$ \\
Platelet count & 200 & $10^{9} / \mathrm{L}$ & $70-300$ \\
$\begin{array}{l}\text { Eosinophilic granulocyte } \\
\text { percentage }\end{array}$ & 1.8 & $\%$ & $0.4-8$ \\
$\begin{array}{l}\text { Neutrophils percentage } \\
\text { Lymphocytes percentage }\end{array}$ & 62.7 & $\%$ & $40-75$ \\
\hline
\end{tabular}

Table 2. Abnormal results of liver function test

\begin{tabular}{lccc}
\hline Project name & Result & Unit & Reference range \\
\hline Alanine aminotransferase & 49 & $\mathrm{U} / \mathrm{L}$ & $7-45$ \\
Aspartate aminotransferase & 64 & $\mathrm{U} / \mathrm{L}$ & $13-40$ \\
Total bilirubin & 147 & $\mu \mathrm{mol} / \mathrm{L}$ & $5.0-21$ \\
Direct bilirubin & 77.7 & $\mu \mathrm{mol} / \mathrm{L}$ & $0-3.4$ \\
Indirect bilirubin & 69.3 & $\mu \mathrm{mol} / \mathrm{L}$ & $1.3-18.7$ \\
Y-glutamyl transferase & 165 & $\mathrm{IU} / \mathrm{L}$ & $7-45$ \\
Total bile salts & 58.1 & $\mu \mathrm{mol} / \mathrm{L}$ & $0.5-10$ \\
Alkaline phosphatase & 284 & $\mathrm{U} / \mathrm{L}$ & $30-120$ \\
\hline
\end{tabular}

Table 3. Abnormal results of tumor markers

\begin{tabular}{lccc}
\hline Project name & Result & Unit & Reference range \\
\hline Sugar antigen protein (CA-50) & 35.47 & $\mathrm{IU} / \mathrm{ml}$ & $0-25$ \\
Carbohydrate antigen 19-9 (CA19-9) & 92.7 & $\mathrm{U} / \mathrm{ml}$ & $0-34$ \\
Ferritin & 422.9 & $\mathrm{ng} / \mathrm{ml}$ & $13-150$ \\
\hline
\end{tabular}

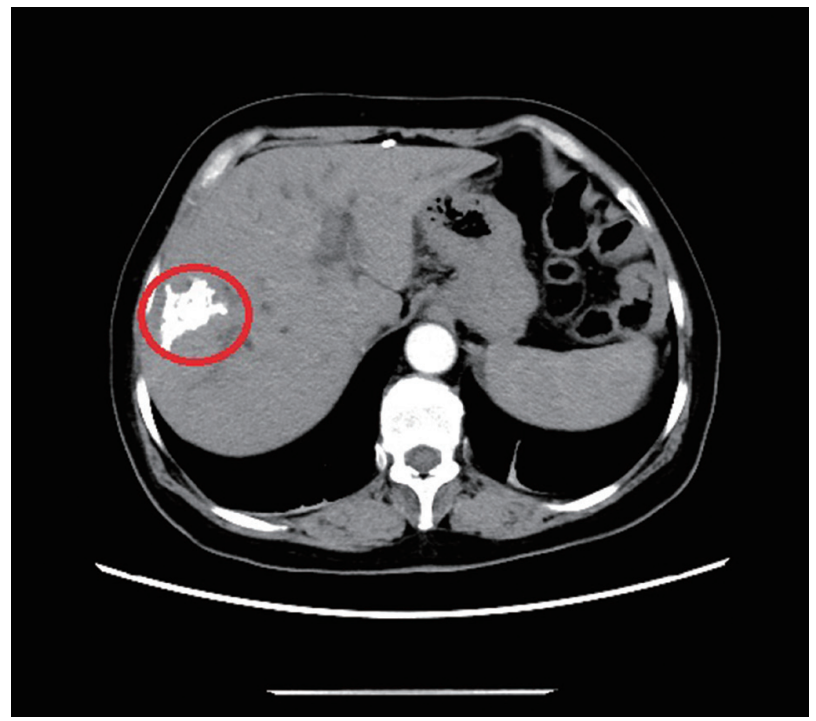

Fig. 1. The abdominal and pelvic CT scan revealed that highdensity nidi were scattered in the right lobe of the liver, approximately $42 \times 22 \mathrm{~mm}$ (red ring marker site).

cholangiocarcinoma and decided to have an operation. We performed radical hepatic hilar cholangiocarcinoma and

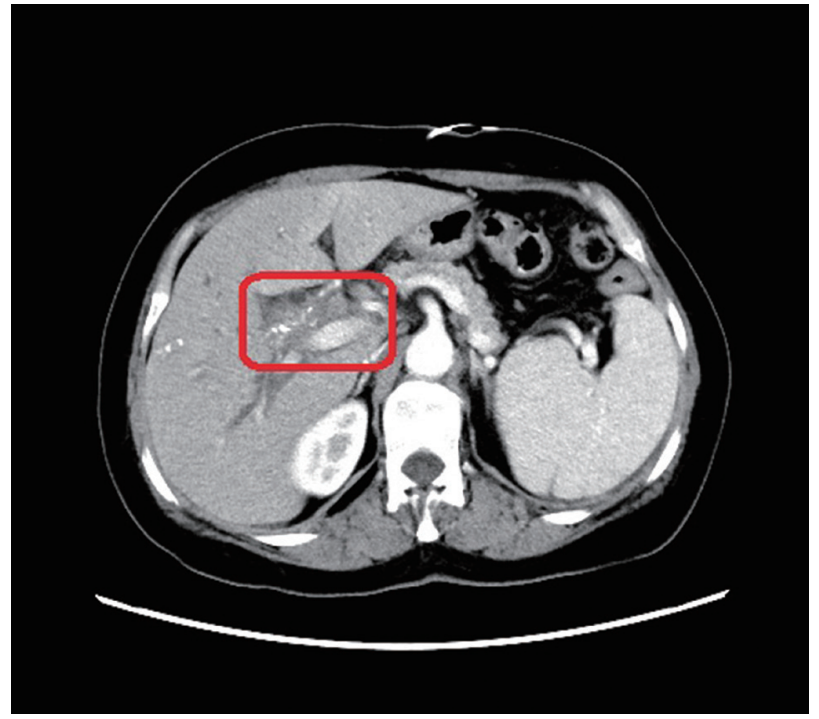

Fig. 2. The abdominal and pelvic CT scan showed that thickening of the bile duct wall in the hilus (red box marker site).

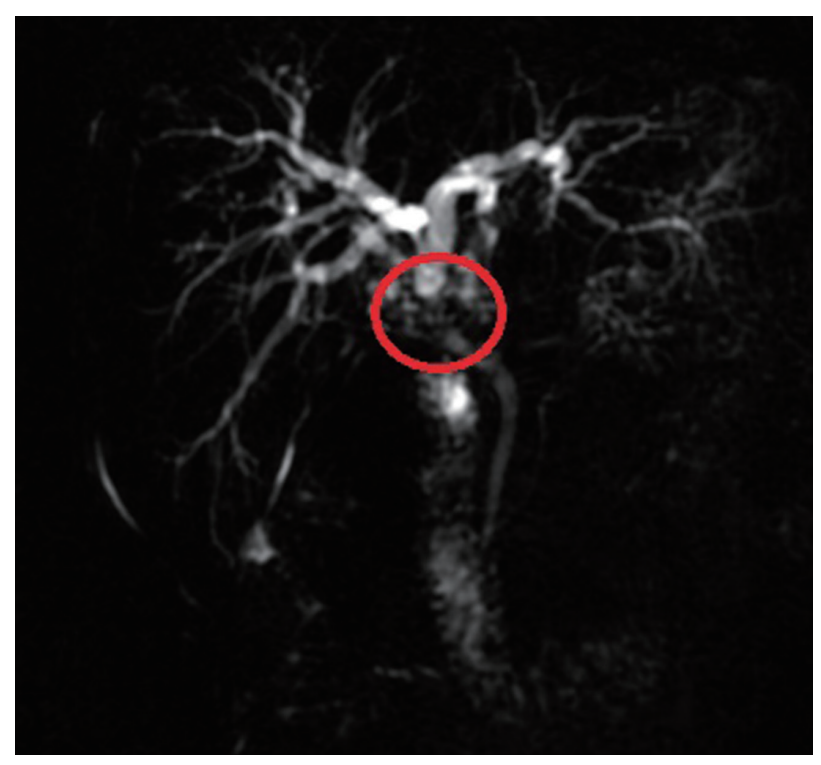

Fig. 3. MRCP indicated local thickening of the upper common bile duct and luminal stenosis (red ring marker site).

Roux-en-Y hepaticojejunostomy under general anesthesia. However, the pathological examination of surgical specimens turned out to be hilar bile duct alveolar echinococcosis. Microscopically, alveolar cysts of varying sizes were observed scattering in bile duct epithelial tissues, with granulation tissues and fibrous tissues proliferation around them. There was necrosis among the vesicles, accompanied by foreign body granuloma formation. The cuticle and germinal layer can be seen in the vesicles of alveolar hydatid (Fig. 4). Furthermore, the patient's 


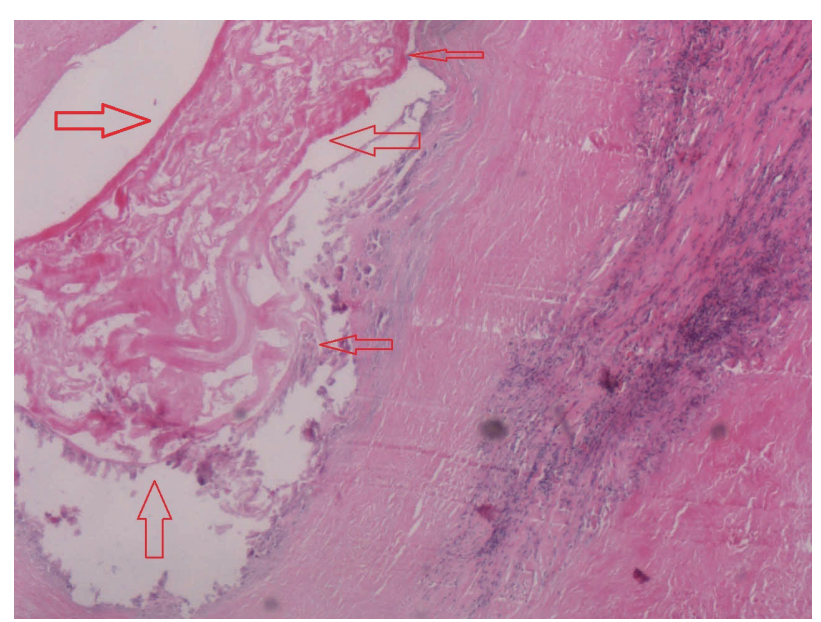

Fig. 4. The pathological examination of surgical specimen manifested that alveolar cysts (the site indicated by the arrows) could be seen in bile duct epithelial tissues (H\&E, × 400).

hydatid-enzyme immunostaining test showed weak positive.

\section{DISCUSSION}

The diagnosis standard of HAE according to WHO-IWGE has the following aspects [5]: (1) Patient's history, living space and contact of cattle that is the source of infection is important; (2) the symptoms such as abdominal distention, pain or discomfort, a mass/masses in hepatic region, jaundice, high fever or dizziness, headache, coughing and other symptoms; (3) The abdominal X-ray is limited in the diagnosis of HAE. Only when the external capsule calcified, $\mathrm{X}$-rays can be seen as a circular or arc-shaped calcification. (4) Ultrasound examination shows strong echo lesions with heterogeneous echoes inside. There is no envelope around the lesion of HAE and the margin is irregular. It can be confused with primary liver cancer, hepatic hemangioma and focal hyperplasia. (5) CT examination shows inhomogeneous solid mass, unclear boundary and no obvious enhancement in enhanced scan. The lesions may be vesicles, calcification, and liquefaction necrosis. (6) MRI examination reveals an irregular solid mass which is mainly lowsignal. After enhancement, the normal tissue is enhanced and the nidus is not strengthened, which makes the boundary clear. (7) Immunological examination is a specific examination index for early diagnosis of HAE, such as ELISA, IHA, DIGFA and so on [6]. (8) Puncture biopsy had been contraindicated in the diagnosis of hydatid disease of the liver, because it could cause anaphylaxis and peritoneal implantation and dissemination. However, the literature demonstrated that puncture biopsy was safe and reliable. In most cases, it was used for small simple hepatic hydatid cysts, especially when hepatic hydatid cysts had a serious impact on the biliary system and needed to be evaluated for biliary tract status [7].

In this case, the patient had typical clinical manifestations of cholangiocarcinoma such as abdominal pain, jaundice, weight loss and his condition did not improve significantly after antiinflammatory treatment. The abdominal and pelvic CT scan and upper abdominal MRI scan both showed symbols leading to diagnosis of cholangiocarcinoma and CT also revealed multiple enlarged retroperitoneal lymph nodes. Besides that, the patient's tumor marker CA19-9 had a certain degree of increase. Based on the evidence above, we diagnosed the patient as cholangiocarcinoma and performed a surgery. However, the postoperative pathology confirmed that it was hilar bile duct alveolar echinococcosis.

Almost all of the HAE originates from the liver. There are 3 ways for extrahepatic metastasis: blood metastasis, direct infiltration and lymph node metastasis. Blood metastasis: when the HAE invades the intrahepatic blood vessels in an invasive way, the larvae enters the blood and proliferates in the way of endogenous reproductive buds and external reproductive buds [8], then the exfoliated buds might transfer to the lung, brain, bone and other parts of the body through circulation. Direct infiltration: the alveolar echinococcosis develops to a mass in the liver and spreads to the surrounding normal liver parenchyma and may even invade the adjacent organs. The right adrenal gland is the most common invaded organ. Lymph node metastasis: lymphatic metastasis of HAE is relatively rare, which is most common in the portal lymph nodes and the retroperitoneal lymph node. Calcification in the lymph nodes has certain diagnostic significance. In this case, the nidus in the right lobe of the liver from the abdominal CT scan showed high-density, which can be easily taken as stone or calcification. After pathologic findings, we considered the high density nidus in the right lobe of the liver might be calcification of hepatic alveolar echinococcosis and the hilar bile duct alveolar echinococcosis might be caused by metastasis of HAE. This case suggests that we should consider differential diagnosis of HAE and cholangiocarcinoma in focal liver lesions.

\section{ACKNOWLEDGMENTS}

Project supports were provided by the Qinghai Provincial Natural Science Foundation (grant no. 2017-ZJ-914). The au- 
thors are very grateful to QingHai Science \& Technology Department for help.

\section{CONFLICT OF INTEREST}

The authors declare no conflict of interest related to this study.

\section{REFERENCES}

1. Parray FQ, Ahmad SZ, Sherwani AY, Chowdri NA, Wani KA. Primary paraspinal hydatid cyst: a rare presentation of echinococcosis. Int J Surg 2010; 8: 404-406.

2. NunnariG, PinzoneMR, Gruttadauria S, Celesia BM, Madeddu G, Malaquarnera G, Pavone P, Cappellani A, Cacopardo B. Hepatic echinococcosis: clinical and therapeuticaspects. World J Gastroenterol 2012; 18: 1448-1458.

3. Kawamura N, Kamiyama T, Sato N, Nakannishi K, Yokoo H,
Kamachi H, Tahara M, Yamaga S, Matsushita M. Long-term results of hepatectomy for patients with alveolar echinococcosis: a singlecenter experience. J Am Coll Surg 2011; 212: 804-812.

4. Parsak CK, Demiryurek HH, Inal M, Sakman G, Koltas IS, Erkocak EU, Korkmaz M, Acarturk TO. Alveolar hydatid disease: imaging findings and surgical approach. Acta Chir Belg 2007; 107: 572-577.

5. Brunetti E, Kern P, Vuitton DA. Expert consensus for the diagnosis and treatment of cystic and alveolar echinococcosis in humans. Acta Trop 2010; 114: 1-16.

6. Zhang W, McManus DP. Recent advances in the immunology and diagnosis of echinococcosis. FEMS Immunol Med Microbiol 2006; 47: 24-41.

7. Men S, Hekimoğlu B, Yücesoy C, Arda IS, Baran I. PercutaneousTreatment of Hepatic hydatid cysts: an alternative to Surgery. AJR Am J Roentgenol 1999; 172: 83-89.

8. Zhou X, Lu G, Yu Z, Gao F, Bian T. Long-term follow-up of whole lung lavage in patients with pulmonary alveolar proteinosis. Exp Ther Med 2014; 8: 763-768. 